\title{
Search for combinations of thermal n-point functions with analytic extensions
}

\author{
H. Arthur Weldon \\ Department of Physics, West Virginia University, Morgantown WV 26506-6315
}

(Dated: December 13, 2018)

\begin{abstract}
The $2^{n}$ different $n$-point functions that occur in real-time thermal field theory are Fourier transformed to real energies. Because of branch cuts in various energy variables, none of these functions can be extended analytically to complex energies. The known linear combinations that form the fully retarded and advanced functions can be extended analytically. It is proven that no other linear combinations have an analytic extension to complex energies.
\end{abstract}

\section{INTRODUCTION}

\section{A. Analyticity at $T=0$}

In zero-temperature field theory the analytic properties of amplitudes is of fundamental importance. Physical scattering amplitudes are functions of real energies but they can be extended analytically into certain regions of the complex energy planes. As a consequence, scattering amplitudes are the boundary values of analytic functions when the energy arguments are real.

Crossing symmetry at zero temperature is a property of amplitudes for different processes that are analytic continuations of each other. Without analyticity there could be no crossing symmetry. In the simplest case of $2 \rightarrow 2$ scattering for equal mass particles, the amplitude is a function of the Lorentz invariant variables $s, t, u$ subject to the constraint $s+t+u=4 m^{2}$. In the direct channel $s>4 m^{2}$ while $t$ and $u$ are negative. In one of the crossed channels $s$ and $u$ are negative but $t>4 m^{2}$. Two properties of the scattering amplitude are important: (1) Starting from real energies in either physical region, the amplitude can be extended analytically into a certain region of the complex energy plane. For example, the direct channel amplitude can be extended analytically into the upper half of the complex $s$ plane but not into the lower half because of a branch cut. Similarly for the cross channel. (2) The location of the branch cuts is such that the direct channel amplitude can be analytically continued to obtain the cross channel amplitude. Consequently, one function of the complex energies, when evaluated at the appropriate real energies, can yield the scattering amplitude for three different physical reactions [1].

\section{B. $T \neq 0$ Background}

The real-time formulation of thermal field theory requires a mixture of time-ordered and anti-time-ordered products that give $2^{n}$ different n-point functions [2, 3, 4]. The Fourier transforms are functions of the external energy-momenta $p_{j}$ :

$$
G_{\alpha_{1} \alpha_{2} \ldots \alpha_{n}}\left(p_{1}, p_{2}, \ldots p_{n}\right) \text {. }
$$

Each suffix $\alpha_{j}$ has two possible values, 1 or 2 . These functions arise naturally in the time-path formulation. [See
Eq. (3.2) below.] However, it is often convenient to employ various linear combinations of these basic functions. Keldysh introduced a different set 5 ] that has been used extensively by Heinz and collaborators [6, 7, 8, 9]. Aurenche and Becherawwy used the transformations which simplify the two-point functions and apply them to the n-point functions and introduced what is known as the $R / A$ basis [10] that has important physical applications 11, 12]. Although it is not Feynman's fault, there is an $F / \bar{F}$ basis that was motivated by zero-temperature Feynman propagators. The algebraic relations between these various choice of bases was clarified in two papers by van Weert et al [13, 14]. The analytic properties of the amplitudes was not treated in these investigations.

The question of analyticity. For each $n$ there are $n$ fully-retarded thermal n-point functions and $n$ fullyadvanced thermal n-point functions, as reviewed in Sec. IV. All of these are linear combinations of the basic functions in Eq. (1.1) and all of these can be analytically extended to certain domains of the complex energies $p_{j}^{0}$. The simplicity of these results leads to the question of whether there are other linear combinations of the basic functions in (1.1) that can be analytically extended to other domains of complex energy.

One can sharpen the question by incorporating the results of Evans 16. He showed that the single n-point function of the imaginary-time formulation can be analytically extended away from the discrete, imaginary energies to various domains of complex energies. For $n=4$ there are 32 thermal 4-point functions that have analytic extensions; the fully retarded and advanced functions account for only 8 of these. For $n=5$ there are new 370 thermal 5-point functions that have analytic extensions; the fully retarded and advanced functions account for only 10 of these. The question addressed here is whether any of these new n-point functions that have analytic extensions can be expressed as linear combination of the basic functions in Eq. (1.1).

Outline. Section II treats the thermal two-point functions and shows why particular linear combinations do have analytic extensions. Section III computes the Fourier transforms of the thermal n-point functions and shows why none of the $2^{n}$ functions can be extended analytically to complex energy. Furthermore, none of these functions is the difference of two analytic functions across a branch cut. Section IV summarizes known re- 
sults about the fully retarded and the fully advanced npoint functions. In particular, the retarded can be analytically extended to a domain in which one $p_{j}^{0}$ has a positive imaginary part and all the others have negative imaginary parts; the advanced can be analytically extended to a domain in which one $p_{j}^{0}$ has a negative imaginary part and all the others have positive imaginary parts. Section $\mathrm{V}$ shows that any $n$-point function that is analytic in a more general domain, i.e. one in which at least two energies have positive imaginary parts and at least two energies have negative imaginary parts, cannot be a linear combination of the real-time thermal $n$-point functions.

\section{II. $n=2$ EXAMPLE}

It is instructive to examine two-point functions in preparation for the more general results in Sec. III-V. Throughout the paper, the thermal average of any operator $\Omega$ will be denoted

$$
\sum_{a} P_{a}\langle a|\Omega| a\rangle
$$

where the summation is over a completes set of energy eigenstates $|a\rangle$ with energy $E_{a}$. Here $P_{a}$ is the thermal probability of state $|a\rangle$ :

$$
P_{a}=e^{-\beta E_{a}} / \operatorname{Tr}\left[e^{-\beta H}\right],
$$

and $\beta$ is the inverse temperature.

\section{A. $G_{11}$ propagator}

The simplest 2-point function is the thermal average of the time-ordered product

$$
G_{11}\left(x_{1}, x_{2}\right)=-i \sum_{a} P_{a}\left\langle a\left|T\left[\phi\left(x_{1}\right) \phi\left(x_{2}\right)\right]\right| a\right\rangle .
$$

The Fourier transform is

$$
\begin{array}{r}
\int d^{4} x_{1} d^{4} x_{2} e^{i\left(p_{1} \cdot x_{1}+p_{2} \cdot x_{2}\right)} G_{11}\left(x_{1}, x_{2}\right) \\
=2 \pi \delta\left(p_{1}^{0}+p_{2}^{0}\right) G_{11}\left(p_{1}, p_{2}\right)
\end{array}
$$

Note that $G_{11}\left(p_{1}, p_{2}\right)$ is proportional to $(2 \pi)^{3} \delta^{3}\left(\mathbf{p}_{1}+\mathbf{p}_{2}\right)$. The Fourier transform of the space dependence will be expressed in terms of

$$
\phi_{j} \equiv \int d^{3} x_{j} e^{-i \mathbf{p}_{j} \cdot \mathbf{x}_{j}} \phi\left(0, \mathbf{x}_{j}\right),
$$

which contains the three-momentum $\mathbf{p}_{j}$. The Fourier transform of the time dependence can be performed using the Hamiltonian evolution:

$$
\phi\left(t_{j}, \mathbf{x}_{j}\right)=e^{i H t_{j}} \phi\left(0, \mathbf{x}_{j}\right) e^{-i H t_{j}} .
$$

The dependence on the energies $p_{j}^{0}$ comes through the retarded resolvents

$$
\mathcal{G}_{j}^{R}=\frac{1}{E_{a}+p_{j}^{0}-H+i \epsilon} .
$$

Working this out easily gives

$$
G_{11}\left(p_{1}, p_{2}\right)=\sum_{a} P_{a}\left[\left\langle a\left|\phi_{1} \mathcal{G}_{1}^{R} \phi_{2}\right| a\right\rangle+\left\langle a\left|\phi_{2} \mathcal{G}_{2}^{R} \phi_{1}\right| a\right\rangle\right]
$$

The spectrum of $H$ is positive, definite. For a fixed value of $E_{a}$, the first term has a semi-infinite branch cut in $p_{1}^{0}$ that extends from some finite energy to $\infty$. Because the range of $E_{a}$ is unbounded when summed over, the first term has a branch cut running the entire length of the real axis, $-\infty<p_{1}^{0}<\infty$. The first term can be extended analytically from real $p_{1}^{0}$ to any complex value with $\operatorname{Im}\left(p_{1}^{0}\right)>0$ without having to cross the branch cut. The second term has a branch cut for $-\infty<p_{2}^{0}<\infty$ and can be extended analytically from real $p_{2}^{0}$ to any complex value satisfying $\operatorname{Im}\left(p_{2}^{0}\right)>0$. Since the complex energies must satisfy $p_{1}^{0}+p_{2}^{0}=0$, these two regions do not overlap. Hence there is no domain of complex energies for which the time-ordered thermal propagator is naturally analytic. The qualifying word 'naturally' is included because the dynamics may allow one of the terms in Eq. 2.5 to be analytically continued through the branch cut.

\section{B. $G_{12}$ propagator}

In real-time thermal field theory there are four types of propagators, labeled $G_{11}, G_{12}, G_{21}, G_{22}$ [2, 3, 4]. The second of these is

$$
G_{12}\left(x_{1}, x_{2}\right)=-i \sum_{a, b} C_{a b}\left\langle a\left|\phi\left(x_{2}\right)\right| b\right\rangle\left\langle b\left|\phi\left(x_{1}\right)\right| a\right\rangle,
$$

where

$$
C_{a b}=e^{\sigma\left(E_{a}-E_{b}\right)} P_{a},
$$

and $0 \leq \sigma \leq \beta$. The Fourier transform is

$$
G_{12}\left(p_{1}, p_{2}\right)=-i e^{-\sigma p_{2}^{0}} \sum_{a} P_{a}\left\langle a\left|\phi_{2} 2 \pi \delta\left[E_{a}+p_{2}^{0}-H\right] \phi_{1}\right| b\right\rangle .
$$

The Dirac delta function forces $p_{2}^{0}$ to be real and therefore $p_{1}^{0}$ is real. No analytic extension is possible.

\section{Linear combination with an analytic extension}

Although neither $G_{11}$ nor $G_{12}$ can be extended analytically to complex energy, there is a simple linear combination of the two that can be extended. This requires the advanced resolvent,

$$
\mathcal{G}_{j}^{A}=\frac{1}{E_{a}+p_{j}^{0}-H-i \epsilon} .
$$


which is related to the retarded resolvent by

$$
\mathcal{G}_{j}^{R}+i 2 \pi \delta\left[E_{a}+p_{j}^{0}-H\right]=\mathcal{G}_{j}^{A} .
$$

The following linear combination of $G_{11}$ and $G_{12}$ changes the $\mathcal{G}_{2}^{R}$ in Eq. (2.5) to $\mathcal{G}_{2}^{A}$ :

$$
\begin{aligned}
& G_{11}\left(p_{1}, p_{2}\right)-e^{\sigma p_{2}^{0}} G_{21}\left(p_{1}, p_{2}\right) \\
& \quad=\sum_{a} P_{a}\left[\left\langle a\left|\phi_{1} \mathcal{G}_{1}^{R} \phi_{2}\right| a\right\rangle+\left\langle a\left|\phi_{2} \mathcal{G}_{2}^{A} \phi_{1}\right| a\right\rangle\right] .
\end{aligned}
$$

The term containing $\mathcal{G}_{1}^{R}$ can be extended analytically to $\operatorname{Im}\left(p_{1}^{0}\right)>0$; the term containing $\mathcal{G}_{2}^{A}$ can be analytically extended to $\operatorname{Im}\left(p_{2}^{0}\right)<0$. Since $p_{1}^{0}+p_{2}^{0}=0$ these two conditions are compatible. This combination is known as the retarded propagator:

$$
R\left(\stackrel{\circ}{p_{1}}, p_{2}\right)=G_{11}\left(p_{1}, p_{2}\right)-e^{\sigma p_{2}^{0}} G_{21}\left(p_{1}, p_{2}\right),
$$

where the circumflex over $p_{1}$ will be used to indicate that $p_{1}^{0}$ is the energy that can have a positive imaginary part.

Starting with Eq. (2.5) there is another way to produce a 2-point function that can be analytically extended, viz. change $\mathcal{G}_{1}^{R}$ to $\mathcal{G}_{1}^{A}$. This produces the advanced two-point function $A\left(p_{1}, p_{2}^{\circ}\right)$ which is analytic for $\operatorname{Im}\left(p_{1}^{0}\right)<0$ and $\operatorname{Im}\left(p_{2}^{0}\right)>0$.

\section{III. n-POINT FUNCTIONS IN THE REAL-TIME FORMULATION}

Thermal n-point functions are defined [2, 3, 4] in the complex time plane on a contour that consists of two parts: $C_{1}$ runs along the real time axis from $-\infty$ to $\infty$; $C_{2}$ runs anti-parallel to the real time axis from $\infty-i \sigma$ to $-\infty-i \sigma$. The parameter $\sigma$ lies in the range $0 \leq \sigma \leq \beta$. A scalar field $\phi(x)$ may be defined on contours $C_{1}$ and $C_{2}$ by introducing

$$
\Phi_{\alpha}(x)=\left\{\begin{array}{cc}
\phi(x) & \text { if } \alpha=1 \\
e^{\sigma H} \phi(x) e^{-\sigma H} & \text { if } \alpha=2
\end{array}\right.
$$

The thermal Green functions are

$$
\begin{aligned}
& G_{\alpha_{1} \alpha_{2} \ldots \alpha_{n}}\left(x_{1}, x_{2}, \ldots x_{n}\right) \\
& \quad=(-i)^{n-1} \sum_{a} P_{a}\left\langle a\left|T_{C}\left[\Phi_{\alpha_{1}}\left(x_{1}\right) \ldots \Phi_{\alpha_{n}}\left(x_{n}\right)\right]\right| a\right\rangle
\end{aligned}
$$

where $T_{C}$ denotes contour ordering. Complex conjugation of a Green function interchanges the indices, $1 \leftrightarrow 2$,

$$
\begin{aligned}
& {\left[G_{\alpha_{1} \alpha_{2} \ldots \alpha_{n}}\left(x_{1}, x_{2}, \ldots x_{n}\right)\right]^{*}} \\
& \quad=(-1)^{n-1} G_{\bar{\alpha}_{1} \bar{\alpha}_{2} \ldots \bar{\alpha}_{n}}\left(x_{1}, x_{2}, \ldots x_{n}\right),
\end{aligned}
$$

where $\overline{1}=2$ and $\overline{2}=1$.

When all the fields are on contour $C_{1}$ the result is the thermal average of the time-ordered product of $n$ fields:

$$
G_{1 \ldots 1}\left(x_{1}, \ldots x_{n}\right)=(-i)^{n-1} \sum_{a} P_{a}\left\langle a\left|T\left[\phi\left(x_{1}\right) \ldots \phi\left(x_{n}\right)\right]\right| a\right\rangle .
$$

When all the fields are on $C_{2}$ the result is thermal average of the anti-time-ordered product:

$$
G_{2 \ldots 2}\left(x_{1}, \ldots x_{n}\right)=(-i)^{n-1} \sum_{a} P_{a}\left\langle a\left|\widetilde{T}\left[\phi\left(x_{1}\right) \ldots \phi\left(x_{n}\right)\right]\right| a\right\rangle,
$$

where $\tilde{T}$ denotes anti-time-ordering.

In the general case, $\ell$ fields are on contour $C_{2}$ and the remaining $n-\ell$ are on contour $C_{1}$. For example, if $x_{1}, x_{2}, \ldots x_{\ell}$ are the coordinates of the fields on $C_{2}$ and $x_{\ell+1}, \ldots x_{n}$ are the coordinates of the fields on $C_{1}$, the contour-ordered Green function is

$$
\begin{array}{r}
\underbrace{G_{n-\ell}^{2 \ldots 2} \underbrace{1 \ldots 1}_{n-\ell}}_{\ell}\left(x_{1}, \ldots x_{n}\right) \\
=(-i)^{n-1} \sum_{a, b} C_{a b}\left\langle a\left|\widetilde{T}\left[\phi\left(x_{1}\right) \ldots \phi\left(x_{\ell}\right)\right]\right| b\right\rangle \\
\quad \times\left\langle b\left|T\left[\phi\left(x_{\ell+1}\right) \ldots \phi\left(x_{n}\right)\right]\right| a\right\rangle,
\end{array}
$$

where $C_{a b}$ is the same as in Eq. (2.7). Because $\sigma$ is in the range $0 \leq \sigma \leq \beta, C_{a b}$ will decrease as either $E_{a}$ or $E_{b}$ become large. Eq. (3.6) is invariant under permutations among the first $\ell$ coordinates and invariant under permutations among the last $n-\ell$ coordinates. Any permutation that interchanges one of the first $\ell$ coordinates with one of the last $n-\ell$ will give a new function.

\section{A. Fourier transforms}

Invariance under time translation guarantees that the Fourier transforms will have the form

$$
\begin{array}{r}
\int \prod_{j=1}^{n}\left[d^{4} x_{j} e^{i p_{j} \cdot x_{j}}\right] G_{\alpha_{1} \alpha_{2} \ldots \alpha_{n}}\left(x_{1}, x_{2}, \ldots x_{n}\right) \\
=2 \pi \delta\left[\sum_{s=1}^{n} p_{s}^{0}\right] G_{\alpha_{1} \alpha_{2} \ldots \alpha_{n}}\left(p_{1}, p_{2}, \ldots p_{n}\right)
\end{array}
$$

As before, $G(p)$ is proportional to a Dirac delta function that conserves three-momentum. The constraint of energy conservation,

$$
p_{1}^{0}+p_{2}^{0}+p_{3}^{0}+\ldots+p_{n}^{0}=0,
$$

will be very important in what follows.

\section{Fourier transform of $G_{11 \ldots 1}$.}

The Fourier transform of Eq. (3.4) can be written as a sum over all permutations:

$$
\begin{aligned}
& G_{1 \ldots 1}\left(p_{1}, \ldots p_{n}\right) \\
& =\sum_{a} \sum_{[\text {perm }]} P_{a}\left\langle a\left|\phi_{[1]} \mathcal{G}_{[1]}^{R} \phi_{[2]} \mathcal{G}_{[12]}^{R} \ldots \phi_{[n]}\right| a\right\rangle,
\end{aligned}
$$


where [ ] is a permutation that sends the ordered set $\{1,2, \ldots, n\}$ into the ordered set $\{[1],[2], \ldots,[n]\}$. The notation is the same as Eqs. (2.1), (2.2), and(2.4). The retarded resolvents depend on the sums of various $p_{j}^{0}$ :

$$
\begin{aligned}
\mathcal{G}_{i j}^{R} & =\frac{1}{E_{a}+p_{i}^{0}+p_{j}^{0}-H+i \epsilon} \\
\mathcal{G}_{i j k}^{R} & =\frac{1}{E_{a}+p_{i}^{0}+p_{j}^{0}+p_{k}^{0}-H+i \epsilon} \\
\mathcal{G}_{i j k \ell}^{R} & =\frac{1}{E_{a}+p_{i}^{0}+p_{j}^{0}+p_{k}^{0}+p_{\ell}^{0}-H+i \epsilon} .
\end{aligned}
$$

Zero-temperature limit. At $T=0$ only the vacuum state $|0\rangle$ contributes to Eq. (3.10). The n-point function is

$$
G_{1 \ldots 1}\left(p_{1}, \ldots p_{n}\right) \rightarrow \sum_{[\text {perm }]}\left\langle 0\left|\phi_{[1]} \mathcal{G}_{[1]}^{R} \phi_{[2]} \mathcal{G}_{[12]}^{R} \ldots \phi_{[n]}\right| 0\right\rangle
$$

but with $E_{a}=0$ in the resolvents:

$$
\mathcal{G}_{z}^{R} \rightarrow \frac{1}{z-H+i \epsilon} .
$$

Since the spectrum of the Hamiltonian is positive, the vacuum resolvent produces no branch cuts when $z$ is negative. For $2 \rightarrow 2$ scattering, two energies $p_{1}^{0}$ and $p_{2}^{0}$ are positive real; two energies $p_{3}^{0}$ and $p_{4}^{0}$ are negative real. Thus at zero temperature there is no problem in analytically extending the 4-point function off the real axis to allow $p_{1}^{0}$ and $p_{2}^{0}$ to lie in the first quadrant and $p_{3}^{0}$ and $p_{4}^{0}$ to lie in the third quadrant.

\section{Fourier transform of $G_{22 \ldots 2}$}

The Green function in which all fields are anti-timeordered is given in Eq. (3.5) and has the Fourier transform

$$
\begin{aligned}
& G_{2 \ldots 2}\left(p_{1}, \ldots p_{n}\right) \\
& =(-1)^{n-1} \sum_{a} \sum_{[\text {perm }]} P_{a}\left\langle a\left|\phi_{[1]} \mathcal{G}_{[1]}^{A} \phi_{[2]} \mathcal{G}_{[12]}^{A} \ldots \phi_{[n]}\right| a\right\rangle,
\end{aligned}
$$

The denominators of the advanced resolvents have a negative imaginary part, $-i \epsilon$ as in Eq. 2.8, whereas the retarded have a positive imaginary part, $+i \epsilon$.

\section{Fourier transform of $G_{2 \ldots 21 \ldots 1}$}

In Eq. (3.6) the times $t_{1}, t_{2}, \ldots t_{\ell}$ are independent of the times $t_{\ell+1}, \ldots t_{n}$ and consequently the Fourier transform of $G_{2 \ldots 21 \ldots 1}$ contains the Dirac delta function

$$
i 2 \pi \delta\left[E_{a}+\sum_{j=1}^{\ell} p_{j}^{0}-E_{b}\right] .
$$

This can be written as the difference between an advanced resolvent and a retarded resolvent:

$$
\left\langle b\left|\mathcal{G}_{12 \ldots \ell}^{A}-\mathcal{G}_{12 \ldots \ell}^{R}\right| b\right\rangle .
$$

The Fourier transform of Eq. (3.6) is

$$
\begin{aligned}
& G_{\ell}^{2 \ldots 2} \underbrace{1 \ldots 1}_{n-\ell}\left(p_{1}, p_{2}, \ldots p_{n}\right)=(-1)^{\ell} e^{-\sigma \sum_{1}^{\ell} p_{j}^{0}} \sum_{a} P_{a} \sum_{\left[\text {perm }^{\prime}\right]} \\
& \left\langle a\left|\left(\phi_{[1]} \mathcal{G}_{[1]}^{A} \phi_{[2]} \mathcal{G}_{[12]}^{A} \ldots \phi_{[\ell]}\right)\left(\mathcal{G}_{12 \ldots \ell}^{A}-\mathcal{G}_{12 \ldots \ell}^{R}\right)\left(\phi_{[\ell+1]} \mathcal{G}_{12 \ldots \ell[\ell+1]}^{R} \phi_{[\ell+2]} \mathcal{G}_{12 \ldots \ell[\ell+1, \ell+2]}^{R} \ldots \phi_{[n]}\right)\right| a\right\rangle,
\end{aligned}
$$

where $\left[\right.$ perm $\left.^{\prime}\right]$ are permutations that do not mix the set $\{1,2, \ldots \ell\}$ with the set $\{\ell+1, \ldots n\}$. A useful relation is

$$
\begin{aligned}
e^{-\beta \sum_{1}^{\ell} p_{j}^{0}} G_{\underbrace{2} \ldots 2} \underbrace{1 \ldots 1}_{n-\ell}\left(p_{1}, p_{2}, \ldots p_{n}\right)=(-1)^{\ell} e^{-\sigma \sum_{1}^{\ell} p_{j}^{0}} \sum_{a} P_{a} \sum_{\left[p_{e r m}^{\prime}\right]} \\
\left\langle a\left|\left(\phi_{[\ell+1]} \mathcal{G}_{[\ell+1]}^{R} \phi_{[\ell+2]} \mathcal{G}_{[\ell+1, \ell+2]}^{R} \ldots \phi_{[n]}\right)\left(\mathcal{G}_{\ell+1 \ldots n}^{A}-\mathcal{G}_{\ell+1 \ldots n}^{R}\right)\left(\phi_{[1]} \mathcal{G}_{[1] \ell+1 \ldots n}^{A} \phi_{[2]} \mathcal{G}_{[12] \ell+1 \ldots n}^{A} \ldots \phi_{[\ell]}\right)\right| a\right\rangle,
\end{aligned}
$$

which follows by using Eq. (3.12) and interchanging the states $|a\rangle \leftrightarrow|b\rangle$. It is important to note that in Eq. (3.13) all the advanced resolvents $\mathcal{G}^{A}$ are associated with external lines of type 2. For example, all the functions $G_{2211}, G_{1122}, G_{1212}, G_{2121}, G_{1221}$, and $G_{2112}$ have two $\mathcal{G}^{A}$ resolvents on the left and two $\mathcal{G}^{R}$ resolvents on the right. Eq. (3.14) has the resolvent pattern reversed.

\section{B. Impossibility of analytic extensions}

1. $n=3$ Example

This simplest way to see the impossibility of analytic extension is to examine a specific case, viz $n=3$. The energies for all the three-point functions are constrained 
by

$$
p_{1}^{0}+p_{2}^{0}+p_{3}^{0}=0 .
$$

$G_{111}$ has no analytic extension. The time-ordered function $G_{111}(x)$ contains $3 !=6$ different time orderings and this leads to the Fourier transform being the sum of six different matrix elements of the type in Eq. (3.10):

$$
\begin{aligned}
& G_{111}\left(p_{1}, p_{2}, p_{3}\right)(3.16) \\
&=\sum P_{a}\langle a| \phi_{1} \mathcal{G}_{1}^{R} \phi_{2} \mathcal{G}_{12}^{R} \phi_{3}+\phi_{1} \mathcal{G}_{1}^{R} \phi_{3} \mathcal{G}_{13}^{R} \phi_{2} \\
&+\phi_{2} \mathcal{G}_{2}^{R} \phi_{1} \mathcal{G}_{12}^{R} \phi_{3}+\phi_{2} \mathcal{G}_{2}^{R} \phi_{3} \mathcal{G}_{23}^{R} \phi_{1} \\
&+\phi_{3} \mathcal{G}_{3}^{R} \phi_{1} \mathcal{G}_{13}^{R} \phi_{2}+\phi_{3} \mathcal{G}_{3}^{R} \phi_{2} \mathcal{G}_{23}^{R} \phi_{1}|a\rangle .
\end{aligned}
$$

The one-particle resolvent $\mathcal{G}_{1}^{R}$ can be analytically extended to $\operatorname{Im}\left(p_{1}^{0}\right)>0 ; \mathcal{G}_{2}^{R}$ can be analytically extended to $\operatorname{Im}\left(p_{2}^{0}\right)>0$; and $\mathcal{G}_{3}^{R}$ can be analytically extended to $\operatorname{Im}\left(p_{3}^{0}\right)>0$. These three requirements are incompatible with the constraint (3.15).

$G_{211}$ has no analytic extension. By Eq. (3.13)

$$
\begin{aligned}
& e^{\sigma p_{1}^{0}} G_{211}\left(p_{1}, p_{2}, p_{3}\right) \\
& =\sum_{a} P_{a}\left\langle a\left|\phi_{1}\left(\mathcal{G}_{1}^{R}-\mathcal{G}_{1}^{A}\right)\left(\phi_{2} \mathcal{G}_{12}^{R} \phi_{3}+\phi_{3} \mathcal{G}_{13}^{R} \phi_{2}\right)\right| a\right\rangle .
\end{aligned}
$$

The difference $\mathcal{G}_{1}^{R}-\mathcal{G}_{1}^{A}=-i 2 \pi \delta\left[E_{a}+p_{1}^{0}-H\right]$ forces $p_{1}^{0}$ to be real. For $p_{1}^{0}$ real, an analytic extension of $\mathcal{G}_{12}^{R}$ would require $\operatorname{Im}\left(p_{2}^{0}\right)>0$; analytic extension of $\mathcal{G}_{13}^{R}$ would require $\operatorname{Im}\left(p_{3}^{0}\right)>0$. These two conditions are incompatible with Eq. (3.15.

$G_{211}$ is not a discontinuity. Although $G_{211}$ can only be defined for real energies, it has the appearance of a discontinuity. Define two functions $E$ and $F$ so that

$$
e^{\sigma p_{1}^{0}} G_{211}\left(p_{1}, p_{2}, p_{3}\right)=E\left(p_{1}, p_{2}, p_{3}\right)-F\left(p_{1}, p_{2}, p_{3}\right)
$$

Although $E$ and $F$ are defined only for real energies, it might be possible to extend them analytically into two different domains. Their difference would then be the discontinuity across the branch cut in $p_{1}^{0}$. The explicit functions are

$$
\begin{aligned}
& E\left(p_{1}, p_{2}, p_{3}\right) \\
& \left.\quad=\sum_{a} P_{a}\langle a| \phi_{1} \mathcal{G}_{1}^{R} \phi_{2} \mathcal{G}_{12}^{R} \phi_{3}+\phi_{1} \mathcal{G}_{1}^{R} \phi_{3} \mathcal{G}_{13}^{R} \phi_{2}\right)|a\rangle ; \\
& F\left(p_{1}, p_{2}, p_{3}\right) \\
& \left.\quad=\sum_{a} P_{a}\langle a| \phi_{1} \mathcal{G}_{1}^{A} \phi_{2} \mathcal{G}_{12}^{R} \phi_{3}+\phi_{1} \mathcal{G}_{1}^{A} \phi_{3} \mathcal{G}_{13}^{R} \phi_{2}\right)|a\rangle .
\end{aligned}
$$

$E(p)$ can be analytically extended to the region in which $\operatorname{Im}\left(p_{1}^{0}\right)>0, \operatorname{Im}\left(p_{1}^{0}+p_{2}^{0}\right)>0$, and $\operatorname{Im}\left(p_{1}^{0}+p_{3}^{0}\right)>0$. The latter two conditions imply $\operatorname{Im}\left(p_{3}^{0}\right)<0$ and $\operatorname{Im}\left(p_{2}^{0}\right)<0$, which are consistent with Eq. (3.15). However, $F(p)$ cannot be analytically extended because $\mathcal{G}_{1}^{A}$ requires $\operatorname{Im}\left(p_{1}^{0}\right)<0 ; \mathcal{G}_{12}^{R}$ requires $\operatorname{Im}\left(p_{1}^{0}+p_{2}^{0}\right)>0$ [which makes $\operatorname{Im}\left(p_{3}^{0}\right)<0$ ]; and $\mathcal{G}_{13}^{R}$ requires $\operatorname{Im}\left(p_{1}^{0}+p_{3}^{0}\right)>0$ [which makes $\left.\operatorname{Im}\left(p_{2}^{0}\right)<0\right]$. It is not possible to have $\operatorname{Im}\left(p_{1}^{0}\right)$, $\operatorname{Im}\left(p_{2}^{0}\right)$, and $\operatorname{Im}\left(p_{3}^{0}\right)$ all negative because of (3.15).
Two more examples. For later purposes it is helpful to display two more of the mixed functions that can be obtained from Eq. (3.13):

$$
\begin{aligned}
& e^{\sigma p_{2}^{0}} G_{121}\left(p_{1}, p_{2}, p_{3}\right) \\
& \quad=\sum_{a} P_{a}\langle a| \phi_{2}\left(\mathcal{G}_{2}^{R}-\mathcal{G}_{2}^{A}\right)\left(\phi_{1} \mathcal{G}_{12}^{R} \phi_{3}+\phi_{3} \mathcal{G}_{23}^{R} \phi_{1}|a\rangle\right. \\
& e^{\sigma p_{3}^{0}} G_{112}\left(p_{1}, p_{2}, p_{3}\right) \\
& \quad=\sum_{a} P_{a}\langle a| \phi_{3}\left(\mathcal{G}_{3}^{R}-\mathcal{G}_{3}^{A}\right)\left(\phi_{1} \mathcal{G}_{13}^{R} \phi_{2}+\phi_{2} \mathcal{G}_{23}^{R} \phi_{1}|a\rangle\right. \\
& e^{\sigma\left(p_{2}^{0}+p_{3}^{0}\right)} G_{122}\left(p_{1}, p_{2}, p_{3}\right) \\
& \quad=\sum_{a} P_{a}\left\langle a\left|\left(\phi_{2} \mathcal{G}_{2}^{A} \phi_{3}+\phi_{3} \mathcal{G}_{3}^{A} \phi_{2}\right)\left(\mathcal{G}_{23}^{A}-\mathcal{G}_{23}^{R}\right) \phi_{1}\right| a\right\rangle .
\end{aligned}
$$

These will be used in Sec. IVA.

\section{Arbitrary $n$}

The observations for $n=3$ apply generally. None of the functions $G_{\alpha_{1} \ldots \alpha_{n}}\left(p_{1}, \ldots p_{n}\right)$ displayed in Eqs. (3.10), (3.11), or (3.13) can be extended analytically to complex energies.

\section{ANALYTICITY OF RETARDED AND ADVANCED n-POINT FUNCTIONS}

It is known that certain simple linear combinations of the $G_{\alpha_{1} \ldots \alpha_{n}}\left(p_{1}, \ldots p_{n}\right)$ can be extended analytically to complex energies. These will be reviewed here. Section $\mathrm{V}$ will show that there are no other linear combinations that can be analytically extended.

\section{A. $\mathbf{n}=3$ Example.}

For the three-point functions, the constraint (3.15) requires that any analytic extension must be to a domain in which at least one energy has a negative imaginary part and at least one has a positive imaginary part. However the signs of $\operatorname{Im}\left(p_{j}^{0}\right)$ must all be consistent with the retarded or advanced nature of the resolvents. For example, if $\operatorname{Im}\left(p_{1}^{0}\right)>0$ the corresponding resolvent must be retarded: $\mathcal{G}_{1}^{A}$. The constraint (3.15) implies that $\operatorname{Im}\left(p_{2}^{0}+p_{3}^{0}\right)<0$ and the complementary resolvent must be advanced: $\mathcal{G}_{23}^{A}$. Choosing $\operatorname{Im}\left(p_{2}^{0}\right)$ and $\operatorname{Im}\left(p_{3}^{0}\right)$ both negative determines all the resolvents as follows:

$$
\begin{aligned}
& \operatorname{Im}\left(p_{1}^{0}\right)>0 \rightarrow \operatorname{Im}\left(p_{2}^{0}+p_{3}^{0}\right)<0 \rightarrow \mathcal{G}_{1}^{R} \text { and } \mathcal{G}_{23}^{A} \\
& \operatorname{Im}\left(p_{2}^{0}\right)<0 \rightarrow \operatorname{Im}\left(p_{1}^{0}+p_{3}^{0}\right)>0 \rightarrow \mathcal{G}_{2}^{A} \text { and } \mathcal{G}_{13}^{R} \\
& \operatorname{Im}\left(p_{3}^{0}\right)<0 \rightarrow \operatorname{Im}\left(p_{1}^{0}+p_{2}^{0}\right)>0 \rightarrow \mathcal{G}_{3}^{A} \text { and } \mathcal{G}_{12}^{R} .
\end{aligned}
$$

The three-point function that is analytic in this domain will be called $R\left(\stackrel{\circ}{p_{1}}, p_{2}, p_{3}\right)$. The circumflex over $p_{1}$ indicates that only $p_{1}^{0}$ can have a positive imaginary part. 
All the resolvents are determined in Eq. (4.1) and give

$$
\begin{aligned}
& R\left(p_{1}^{\circ}, p_{2}, p_{3}\right) \\
& =\sum P_{a}\langle a| \phi_{1} \mathcal{G}_{1}^{R} \phi_{2} \mathcal{G}_{12}^{R} \phi_{3}+\phi_{1} \mathcal{G}_{1}^{R} \phi_{3} \mathcal{G}_{13}^{R} \phi_{2} \\
& +\phi_{2} \mathcal{G}_{2}^{A} \phi_{1} \mathcal{G}_{12}^{R} \phi_{3}+\phi_{2} \mathcal{G}_{2}^{A} \phi_{3} \mathcal{G}_{23}^{A} \phi_{1} \\
& +\phi_{3} \mathcal{G}_{3}^{A} \phi_{1} \mathcal{G}_{13}^{R} \phi_{2}+\phi_{3} \mathcal{G}_{A}^{R} \phi_{2} \mathcal{G}_{23}^{A} \phi_{1}|a\rangle .
\end{aligned}
$$

This can be expressed as a linear combination of the $G_{\alpha_{1} \alpha_{2} \alpha_{3}}$ as follows. To change $G_{111}\left(p_{1}, p_{2}, p_{3}\right)$ in Eq. (3.16) into (4.2), the first step is to change $\mathcal{G}_{2}^{R}$ into $\mathcal{G}_{2}^{A}$. This can be done by subtracting Eq. (3.18) from Eq. (3.16):

$$
\begin{aligned}
G_{111}\left(p_{1}, p_{2}, p_{3}\right)- & e^{\sigma p_{2}^{0}} G_{121}\left(p_{1}, p_{2}, p_{3}\right) \\
=\sum P_{a}\langle a| \phi_{1} \mathcal{G}_{1}^{R} \phi_{2} \mathcal{G}_{12}^{R} \phi_{3}+\phi_{1} \mathcal{G}_{1}^{R} \phi_{3} \mathcal{G}_{13}^{R} \phi_{2} & +\phi_{2} \mathcal{G}_{2}^{A} \phi_{1} \mathcal{G}_{12}^{R} \phi_{3}+\phi_{2} \mathcal{G}_{2}^{A} \phi_{3} \mathcal{G}_{23}^{R} \phi_{1} \\
& +\phi_{3} \mathcal{G}_{3}^{R} \phi_{1} \mathcal{G}_{13}^{R} \phi_{2}+\phi_{3} \mathcal{G}_{3}^{R} \phi_{2} \mathcal{G}_{23}^{R} \phi_{1}|a\rangle
\end{aligned}
$$

Next subtract $e^{\sigma p_{3}^{0}} G_{112}\left(p_{1}, p_{2}, p_{3}\right)$ in order to change $\mathcal{G}_{3}^{R}$ into $\mathcal{G}_{3}^{A}$. Then add $e^{\sigma\left(p_{2}^{0}+p_{3}^{0}\right)} G_{122}$ to change $\mathcal{G}_{23}^{R}$ into $\mathcal{G}_{23}^{A}$. This gives the retarded function as a linear combination of the $G_{\alpha_{1} \alpha_{2} \alpha_{3}}$ :

$$
\begin{aligned}
R\left(p_{1}^{\circ}, p_{2}, p_{3}\right)= & G_{111}\left(p_{1}, p_{2}, p_{3}\right)-e^{\sigma p_{2}^{0}} G_{121}\left(p_{1}, p_{2}, p_{3}\right) \\
& -e^{\sigma p_{3}^{0}} G_{112}\left(p_{1}, p_{2}, p_{3}\right) \\
& +e^{\sigma\left(p_{2}^{0}+p_{3}^{0}\right)} G_{122}\left(p_{1}, p_{2}, p_{3}\right)
\end{aligned}
$$

It is worth emphasizing that none of the $G_{\alpha_{1} \alpha_{2} \alpha_{3}}$ on the right hand side can be extended analytically to complex energies. However the combination on the right hand side can be extended analytically to the region given in Eq. (4.1).

\section{B. Retarded n-point functions and their analytic extension}

Eq. (4.3) is a special case of known results [13, 14]. The n-point retarded function can be expressed as the following linear combination

$$
\begin{aligned}
R\left(\stackrel{\circ}{p_{1}}, p_{2}, \ldots p_{n}\right) & =\sum_{\alpha_{2}=1}^{2} \sum_{\alpha_{3}=1}^{2} \ldots \sum_{\alpha_{n}=1}^{2} \\
\times & C_{\alpha_{2} \ldots \alpha_{n}}^{R} G_{1 \alpha_{2} \alpha_{3} \ldots \alpha_{n}}\left(p_{1}, p_{2}, \ldots p_{n}\right),
\end{aligned}
$$

where the coefficients are

$$
C_{\alpha_{2} \ldots \alpha_{n}}^{R}=\prod_{j=2}^{n}\left[-e^{\sigma p_{j}^{0}}\right]^{\alpha_{j}-1} .
$$

The Fourier transform has the structure

$$
R\left(\stackrel{\circ}{p}_{1}, p_{2}, \ldots p_{n}\right)=\sum_{S=1}^{n !} R_{S} .
$$

Each $R_{S}$ is a thermal average of a particular permutation of the fields $\phi_{1}, \phi_{2}, \ldots \phi_{n}$ of the form

$$
R_{S}=\sum_{a} P_{a}\left\langle a\left|\phi_{i} \mathcal{G}^{A} \phi_{j} \mathcal{G}^{A} \ldots \phi_{k} \mathcal{G}^{A} \stackrel{\downarrow}{\phi_{1}} \mathcal{G}^{R} \phi_{l} \mathcal{G}^{R} \ldots \mathcal{G}^{R} \phi_{m}\right| a\right\rangle .
$$

Wherever $\phi_{1}$ occurs in the permutation, all the resolvents to the left are advanced $\mathcal{G}^{A}$ and all the resolvents to the right are retarded $\mathcal{G}^{R}$. The energy dependence of each resolvents is cumulative from left to right. [See Eq. (4.2).]

Analytic extension of the n-point retarded function. All $n$ ! terms in Eq. (4.5) can be extended analytically to the complex energy domain such that $p_{1}^{0}$ has a positive imaginary part and all the other energies $p_{2}^{0}, p_{3}^{0}, \ldots p_{n}^{0}$ have negative imaginary parts. To verify this statement requires two steps.

(i) The first step is to confirm that the signs of the imaginary parts of all the subenergies are determined. Any partial sum $p_{c}^{0}+p_{d}^{0}+\ldots p_{r}^{0}$ may or may not include $p_{1}^{0}$. If it does not include $p_{1}^{0}$ then the imaginary part of the partial sum is automatically negative $\operatorname{since} \operatorname{Im}\left(p_{1}^{0}\right)>0$. If the partial sum does include $p_{1}^{0}$, then the imaginary part of the partial sum is automatically positive because the omitted $p_{j}^{0}$ has a negative imaginary part.

(ii) The second step is to confirm that every $R_{S}$ can be analytically extended to this domain. In $R_{S}$ the advanced resolvents $\mathcal{G}^{A}$ all depend on partial sums of the energies $p_{i}^{0}+p_{j}^{0}+\ldots p_{m}^{0}$ that do not contain $p_{1}^{0}$ and therefore these advanced resolvents can be extended analytically to the lower half-plane as described in (i). In $R_{S}$ the retarded resolvents $\mathcal{G}^{R}$ all depend on partial sums of the energies $p_{i}^{0}+p_{j}^{0}+\ldots p_{m}^{0}$ that do contain $p_{1}^{0}$ and therefore these advanced resolvents can be extended analytically to the upper half-plane as described in (i).

\section{Advanced n-point functions and their analytic extension}

In a parallel fashion the advanced n-point functions are also linear combinations of the $G_{1 \alpha_{2} \alpha_{3} \ldots \alpha_{n}}$ as follows:

$$
\begin{aligned}
A\left(p_{1}, \stackrel{\circ}{p_{2}}, \stackrel{\circ}{p_{3}}, \ldots \stackrel{\circ}{p_{n}}\right)=\sum_{\alpha_{2}=1}^{2} \sum_{\alpha_{3}=1}^{2} \ldots \sum_{\alpha_{n}=1}^{2} \\
\times C_{\alpha_{2} \ldots \alpha_{n}}^{A} G_{1 \alpha_{2} \alpha_{3} \ldots \alpha_{n}}\left(p_{1}, p_{2}, \ldots p_{n}\right),
\end{aligned}
$$

where now the coefficients are

$$
C_{\alpha_{2} \ldots \alpha_{n}}^{A}=\prod_{j=2}^{n}\left[-e^{(\sigma-\beta) p_{j}^{0}}\right]^{\alpha_{j}-1} .
$$

Eq. (3.14) is useful here. The Fourier transform of the advanced function is a sum of thermal averages of $n$ ! terms:

$$
A\left(p_{1}, \stackrel{\circ}{p_{2}}, \ldots \stackrel{\circ}{p_{n}}\right)=\sum_{S=1}^{n !} A_{S} .
$$


Each $A_{S}$ is a particular permutation of the $\phi$ 's:

$$
A_{S}=\sum_{a} P_{a}\left\langle a\left|\phi_{i} \mathcal{G}^{R} \phi_{j} \mathcal{G}^{R} \ldots \phi_{k} \mathcal{G}^{R} \stackrel{\downarrow}{\phi_{1}} \mathcal{G}^{A} \phi_{l} \mathcal{G}^{A} \ldots \mathcal{G}^{A} \phi_{m}\right| a\right\rangle .
$$

To the left of $\phi_{1}$ all the resolvents are retarded and to the right of $\phi_{1}$ all the resolvents are advanced. The energy dependence of each resolvents is cumulative from left to right.

Analytic extension of the n-point advanced function. All $n$ ! terms in Eq. (4.5) can be extended analytically to the complex energy domain such that $p_{1}^{0}$ has a negative imaginary part and all the other energies $p_{2}^{0}, p_{3}^{0}, \ldots p_{n}^{0}$ have positive imaginary parts. The proof is similar to the retarded case.

\section{FAILURE OF OTHER COMBINATIONS TO HAVE ANALYTIC EXTENSIONS}

The retarded and advanced functions of Sec. IV show that it is possible to form linear combinations of the $G_{\alpha_{1} \ldots \alpha_{n}}\left(p_{1}, \ldots p_{n}\right)$ that can be analytically extended to certain domains of complex energy. The simplicity of those results may suggest that other linear combinations can be analytically extended to other domains of complex energy. This section will demonstrate that there are no other such combinations.

The key feature is the pattern of resolvents. In $G_{111 \ldots 111}$ the resolvents are all retarded as in Eq. (3.10). In $G_{222 \ldots 222}$ the resolvents are all advanced as in Eq. (3.11). In $G_{2 \ldots 21 \ldots 1}$ the resolvents are either a string of advanced followed by a string of retarded as in Eq. (3.13) or a string of retarded followed by a string of advanced as in Eq. (3.14). These possibilities are summarized by

$$
\begin{array}{ll}
G_{111 \ldots 111} & \mathcal{G}^{R} \mathcal{G}^{R} \mathcal{G}^{R} \ldots \mathcal{G}^{R} \\
G_{222 \ldots 222} & \mathcal{G}^{A} \mathcal{G}^{A} \mathcal{G}^{A} \ldots \mathcal{G}^{A} \\
G_{2 \ldots 21 \ldots 1} & \mathcal{G}^{A} \ldots \mathcal{G}^{A} \mathcal{G}^{R} \ldots \mathcal{G}^{R} \text { or } \mathcal{G}^{R} \ldots \mathcal{G}^{R} \mathcal{G}^{A} \ldots \mathcal{G}^{A} .
\end{array}
$$

All $n$ ! terms in the retarded n-point functions in Eq. (4.5) and in the advanced n-point functions in Eq. (4.6) have resolvent patterns of these types.

The retarded and advanced functions are the exception. No other thermal n-point function that can be analytically extended to some domain of complex energy can be expressed as a linear combination of the sequences in Eq. (5.1). The proof relies on the fact that any n-point function that can be analytically extended is a sum of $n$ ! terms

$$
M\left(p_{1}, p_{2}, \ldots p_{n}\right)=\sum_{S=1}^{n !} M_{S} .
$$

Each $M_{S}$ involves one permutation of the $\phi_{1}, \phi_{2}, \ldots \phi_{n}$ and is of the form

$$
M_{S}=\sum_{a} P_{a}\left\langle a\left|\phi_{i} \mathcal{G}_{i} \phi_{j} \mathcal{G}_{i j} \phi_{k} \mathcal{G}_{i j k} \ldots \phi_{r}\right| a\right\rangle
$$

The energies in the resolvents, denoted by the subscripts, are cumulative from left to right. Each resolvent is either retarded or advanced and that determination is the difficult part of the problem. (A simple way to verify this structure is to extend the imaginary-time n-point function from discrete, imaginary energies to continuous complex energies in various domains as discussed in [16].)

The following argument will show that if Eq. (5.2) is analytic when two or more energies have positive imaginary parts and two or more energies have negative imaginary parts, then it cannot be a linear combination of the basis functions in Sec. III.

Suppose that $p_{1}^{0}$ and $p_{2}^{0}$ have positive imaginary parts; $p_{3}^{0}$ and $p_{4}^{0}$ have negative imaginary parts; and $p_{5}^{0}, \ldots p_{n}^{0}$ have imaginary parts of either sign. This certainly does not specify the location of all the subenergies in the complex plane, but it is all that will be necessary. Define two sums of $n-1$ energies:

$$
\begin{aligned}
\zeta & =\sum_{j \neq 2} p_{j}^{0} \\
\eta & =\sum_{j \neq 4} p_{j}^{0} .
\end{aligned}
$$

The relation $p_{2}^{0}+\zeta=0$ implies that $\operatorname{Im}(\zeta)<0$; the relation $p_{4}^{0}+\eta=0$ implies that $\operatorname{Im}(\eta)>0$. To construct an n-point function that can be analytically extended, all the resolvents with energy $\zeta$ must be advanced, viz. $\mathcal{G}_{\zeta}^{A}$; all the resolvents with energy $\eta$ must be retarded, viz. $\mathcal{G}_{\eta}^{R}$.

Among the $n$ ! different orderings of the $\phi_{j}$, there will be terms which begin with $\phi_{1}$ followed by $\phi_{3}$ and that end with $\phi_{4}$. When the appropriate resolvents are included, the thermal averages have the form

$$
\sum_{a} P_{a}\left\langle a\left|\phi_{1} \mathcal{G}_{1}^{R} \phi_{2} \mathcal{G}_{13}^{X} \ldots \phi_{3} \ldots \mathcal{G}_{\eta}^{R} \phi_{4}\right| a\right\rangle,
$$

where $X$ is either retarded or advanced, depending upon the imaginary part of $p_{1}^{0}+p_{3}^{0}$. Another important class of terms are those that begin with $\phi_{3}$ followed by $\phi_{1}$ and end with $\phi_{2}$. These give

$$
\sum_{a} P_{a}\left\langle a\left|\phi_{3} \mathcal{G}_{3}^{A} \phi_{1} \mathcal{G}_{13}^{X} \ldots \phi_{4} \ldots \mathcal{G}_{\zeta}^{A} \phi_{2}\right| a\right\rangle .
$$

The fact that $p_{1}^{0}$ and $p_{2}^{0}$ have positive imaginary parts and that $p_{3}^{0}$ and $p_{4}^{0}$ have negative imaginary parts has determined the resolvents $\mathcal{G}_{1}^{R}, \mathcal{G}_{\eta}^{R}, \mathcal{G}_{3}^{A}$, and $\mathcal{G}_{\zeta}^{A}$. The value of $X$ in $\mathcal{G}_{13}^{X}$ is not yet determined.

Case 1. If $\operatorname{Im}\left(p_{1}^{0}+p_{3}^{0}\right)>0$ then $X=R$ in both equations. In Eq. (5.5) the resolvents are thus in the order $\mathcal{G}_{3}^{A} \mathcal{G}_{13}^{R} \ldots \mathcal{G}_{\zeta}^{A}$. In other words, at least one retarded resolvent occurs in a sequence that begins and ends with advanced resolvents. This sequence does not appear in the list (5.1) and cannot be produced by linear combinations of the basis functions in Eqs. (3.10), (3.11). (3.13), or (3.14). 
Case 2. If $\operatorname{Im}\left(p_{1}^{0}+p_{2}^{0}\right)<0$ then $X=A$ in both equations. In Eq. (5.4) the resolvents are in the or$\operatorname{der} \mathcal{G}_{1}^{R} \mathcal{G}_{13}^{A} \ldots \mathcal{G}_{\eta}^{R}$. In other words, at least one advanced resolvent occurs in a sequence that begins and ends with retarded resolvents. This pattern cannot be constructed out of linear combinations of the basis functions. This concludes the proof.
[1] R.J. Eden, P.V. Landshoff, D.I. Olive, J.C. Polkinghorne, The Analytic S-Matrix (Cambridge Univ. Press, Cambridge, England, 1966).

[2] N.P. Landsman and Ch. G. van Weert, Phys. Rep. 145, 141 (1987).

[3] M. Le Bellac, Thermal Field Theory (Cambridge Univ. Press, Cambridge, Eng., 1996).

[4] A. Das, Finite Temperature Field Theory (World Scientific, Singapore, 1997).

[5] L.V. Keldysh, Sov. Phys. JETP 20, 1018 (1965).

[6] D. Hou, E. Wang, and U. Heinz, J. Phys. G 24, 1861 (1998).

[7] H. Defu, M.E. Carrington, R. Kobes, and U. Heinz, Phys. Rev. D 61, 085013 (2000).

[8] E. Wang and U. Heinz, Phys. Rev. D 66, 025008 (2002).
[9] F. Guerin, hep-ph/0105313

[10] P. Aurenche and T. Becherrawy, Nucl. Phys. B379, 259 (1992).

[11] F.T. Brandt, A. Das, J. Frenkel, and A.J. da Silva, Phys. Rev. D 59, 065004 (1999).

[12] F.T. Brandt, A. Das, and J. Frenkel, Phys. Rev. D 60, 105008 (1999).

[13] M.A.. van Eijck and Ch. G. van Weert, Phys. Lett. B 278, 305 (1992).

[14] M.A. van Eijck, R. Kobes, and Ch. G. van Weert, Phys. Rev. D 50, 4097 (1994).

[15] J.I. Kapusta, Finite Temperature Field Theory (Cambridge Univ. Press, Cambridge, Eng., 1989).

[16] T.S. Evans, Nucl. Phys. B374, 340 (1992), Eq. (5.11). 